\title{
Robust penetrating microelectrodes for neural interfaces realized by titanium micromachining
}

\author{
Patrick T. McCarthy $•$ Kevin J. Otto • Masaru P. Rao
}

Published online: 1 March 2011

(C) The Author(s) 2011. This article is published with open access at Springerlink.com

\begin{abstract}
Neural prosthetic interfaces based upon penetrating microelectrode devices have broadened our understanding of the brain and have shown promise for restoring neurological functions lost to disease, stroke, or injury. However, the eventual viability of such devices for use in the treatment of neurological dysfunction may be ultimately constrained by the intrinsic brittleness of silicon, the material most commonly used for manufacture of penetrating microelectrodes. This brittleness creates predisposition for catastrophic fracture, which may adversely affect the reliability and safety of such devices, due to potential for fragmentation within the brain. Herein, we report the development of titanium-based penetrating microelectrodes that seek to address this potential future limitation. Titanium provides advantage relative to silicon due to its superior fracture toughness, which affords potential for creation of robust devices that are resistant to catastrophic
\end{abstract}

\section{P. T. McCarthy}

School of Mechanical Engineering, Birck Nanotechnology Center, Purdue University,

West Lafayette, IN 47907, USA

K. J. Otto

School of Biological Sciences and the Weldon School of

Biomedical Engineering, Purdue University,

West Lafayette, IN 47907, USA

M. P. Rao

School of Mechanical Engineering, Birck Nanotechnology Center, Purdue University,

West Lafayette, IN 47907, USA

M. P. Rao $(\bowtie)$

Department of Mechanical Engineering,

University of California-Riverside,

A313 Bourns Hall,

Riverside, CA 92521, USA

e-mail: mprao@engr.ucr.edu failure. Realization of these devices is enabled by recently developed techniques which provide opportunity for fabrication of high-aspect-ratio micromechanical structures in bulk titanium substrates. Details are presented regarding the design, fabrication, mechanical testing, in vitro functional characterization, and preliminary in vivo testing of devices intended for acute recording in rat auditory cortex and thalamus, both independently and simultaneously.

Keywords MEMS · Prosthesis · Electrophysiology · Recording $\cdot$ Microstimulation $\cdot$ Brain-machine

\section{Introduction}

Penetrating microelectrode devices, which allow direct and selective recording and stimulation of neural tissues, have provided unprecedented opportunity for enhancing understanding of neurophysiological processes underlying many aspects of human function and behavior (Bak et al. 1990, Gelbard-Sagiv et al. 2008). They have also shown immense promise for restoring neurological functions lost to disease, stroke, or injury (Normann 2007), as exemplified by neural prostheses that have enabled restoration of rudimentary auditory perception (Otto et al. 2008, Otto, Rousche and Kipke 2005a, Otto, Rousche and Kipke 2005b) and control of assistive instrumentation for those with motor dysfunction (Hochberg et al. 2006). However, this technology is still in its formative stages and many challenges remain.

While the improvement of chronic recording reliability remains a key near-term challenge for this emerging technology (Vetter et al. 2004), its eventual maturation into a clinically viable technique for restoring lost neurological function will also require development of devices that are capable of withstanding surgical manipulation by neuro- 
surgeons (Kipke et al. 2008), and are immune to safety hazards associated with fracture-based fragmentation. The intrinsic brittleness of silicon, the material most commonly used for the manufacture of such devices, may ultimately prove limiting in this regard. This brittleness, which arises from the low fracture toughness of silicon, creates nonnegligible probability for catastrophic failure by fracture. This has not been a significant concern in the evolution of this technology thus far. However, the potential for device fragmentation within the brain, no matter how small, coupled with the potentially severe consequences associated with such fragmentation in future clinical settings, suggests need for consideration of means for addressing this potential concern.

In addition to potential safety concerns, propensity for fracture of silicon-based microelectrodes may also adversely affect reliability, since fracture often results in complete loss of device functionality. It also places practical constraint on many current silicon devices with regard to the device length that can be reliably inserted, since risk of buckling-related fracture increases with device length, while opportunity for increasing buckling resistance through increased shank cross section area is constrained by potential adverse affects associated with increased insertion damage (Szarowski et al. 2003, Seymour and Kipke 2007). In many animal models, this practical length limitation hampers access to sub-cortical structures and largely precludes extension towards simultaneous recording within precisely-defined cortical and sub-cortical regions. This latter capability is of particular interest, since it may serve as a critical enabler for enhancing understanding of important neural processing networks, such as the corticothalamic loops that underlie auditory, visual, and somatosensory processing (Nicolelis et al. 1995, Fanselow and Nicolelis 1999, Sherman and Guillery 2002, Winer and Larue 1987, Hubel and N. 1972, Nicolelis and Shuler 2001). This, therefore, provides additional impetus to develop penetrating microelectrode devices with greater robustness.

Alternative microelectrode materials systems that have been explored include those based on ceramics (Moxon et al. 2004b), polymers (Lee et al. 2004a, Schuettler et al. 2005, Stieglitz et al. 2000, Rousche et al. 2001), and metals (Motta and Judy 2005, Fofonoff et al. 2004). Ceramics offer only limited benefit relative to silicon, with regard to reliability and safety, since they suffer from similar or greater propensity for fracture (Ward et al. 2009). Recently reported microelectrodes based on polycrystalline diamond show potential for realization of highly flexible, thin cross section devices with sufficient stiffness for cortical penetration (Chan et al. 2009). However, low signal to noise ratio observed during neural recording indicates need for further device refinement. Polymers used thus far possess sufficient toughness to mitigate fracture. However, their low elastic moduli typically require trade-off between device stiffness and functional reliability, since relatively large cross section areas are required to ensure insertion reliability and recording site placement accuracy (Lee et al. 2004b, Suzuki, Mabuchi and Takeuchi 2003, Takeuchi et al. 2004), which increases insertion damage. A number of strategies have been proposed to circumvent this trade-off, including those based on stiffening through incorporation of dissolvable rigid polymer coatings (Suzuki et al. 2003) or internal reinforcements (Lee et al. 2004b, Takeuchi et al. 2005, Takeuchi et al. 2004).

Metals show particular promise for use in microelectrode applications due to their high fracture toughness, which ensures graceful failure via plastic deformation, rather than catastrophic fracture (i.e. devices will bend before they break). This increases safety by minimizing potential for fragmentation, and may also enhance reliability, since potential exists for maintaining device functionality despite overloading. Moreover, when coupled with the moderate to high moduli of metals, this enables realization of robust microelectrodes with small cross section areas, thus facilitating insertion and reducing injury. However, despite these advantageous characteristics, metallic microelectrodes reported to-date suffer from limitations that may constrain their ultimate safety or utility. For example, in the devices demonstrated by Motta and Judy (Motta and Judy 2005), gold coatings were implemented to prevent exposure of the underlying nickel structural backbone to the physiological environment. This may adversely affect safety, due to potential for cytotoxic $\mathrm{Ni}$ ion release in the event of coating failure. In the devices demonstrated by Fofonoff et al. (Fofonoff et al. 2004), toxicity concerns were largely alleviated through the use of titanium as a structural backbone. However, opportunity for probing sequentially interconnected neural processing networks with such devices is constrained by reliance upon the Utah Electrode Array geometry, which consists of arrayed electrodes with only single, tip-located recording sites.

We have recently reported preliminary results from the development of titanium-based penetrating microelectrodes that seek to address the above-mentioned limitations (McCarthy et al. 2009). Realization of these devices was fundamentally enabled by recently developed techniques that allow, for the first time, fabrication of high-aspect-ratio micromechanical devices within bulk titanium substrates (Aimi et al. 2004, Parker et al. 2005). Herein, we expand upon this report to detail the design, fabrication, mechanical testing, and in vitro functional characterization of titaniumbased microelectrode devices intended for use in acute rat auditory cortex and thalamus recording, both independently and simultaneously. Results from acute in vivo characterization of these devices are also briefly touched upon, but 
are more fully elaborated elsewhere (McCarthy, Rao and Otto 2011).

\section{Design and fabrication}

\subsection{Design}

The microelectrodes produced in this effort were designed to study auditory cortex and thalamus responses within a rat model, both individually and simultaneously. As such, short devices ( $2 \mathrm{~mm}$ shank length) were designed for acute cortical recording, and longer devices $(4.9 \& 5.4 \mathrm{~mm}$ shank lengths) were designed for acute thalamic recording, independently of, or in conjunction with cortex. Both device types were modeled after commercially-available "Michigan" type electrode arrays (A1×16-5 mm 100-413, NeuroNexus Technologies, Ann Arbor, MI; Shank length = $5 \mathrm{~mm}$, shank thickness $\sim 15 \mu \mathrm{m}$, shank width $=33-200 \mu \mathrm{m}$ (distal \& proximal ends of shank, respectively), recording site diameter $=23 \mu \mathrm{m}, \&$ recording site pitch $=100 \mu \mathrm{m}$ ). Each titanium device contained 16 independently addressable circular recording sites, and 18 distinct design variants were developed with differing shank length $(2,4.9$, \& $5.4 \mathrm{~mm})$, recording site diameter $(23 \& 40 \mu \mathrm{m})$, and pitch $(50,75,100, \& 150 \mu \mathrm{m})$. Since optimal designs for the intended applications have yet to be defined, the given variation of these parameters was reflective of the desire to eventually determine the most favorable combination that ensures consistent sampling of discrete neurons within the targeted neural regions with high signal to noise ratio.

Figures 1 and 2 show schematic representations of device design variants for acute auditory cortical and corticothalamic recording, respectively. In all devices, recording sites were connected by $5 \mu \mathrm{m}$ wide traces (with $9 \mu \mathrm{m}$ pitch) to remote $100 \mu \mathrm{m} \times 100 \mu \mathrm{m}$ contact pads (with $150 \mu \mathrm{m}$ pitch) arrayed at the proximal end of the device. In devices with $23 \mu \mathrm{m}$ diameter recording sites, this resulted in shanks with width that tapered from $48 \mu \mathrm{m}$ at the recording site closest to the distal end of the shank (i.e. near the sharp tip) to $192 \mu \mathrm{m}$ at the base. In the $40 \mu \mathrm{m}$ diameter recording site devices, shank width ranged from 65 to $209 \mu \mathrm{m}$. Devices intended for cortical recording were designed with 16 recording sites arrayed from the distal end of the shank. Devices intended for corticothalamic recording were designed with one set of 8 recording sites arrayed within the vicinity of $2 \mathrm{~mm}$ from the proximal end of the shank, and an additional set of 8 recording sites arrayed from the distal end of the shank.

\subsection{Fabrication}

The titanium-based microelectrode devices were fabricated from $25.4 \pm 7.62 \mu \mathrm{m}$ thick Ti foil substrates (Gr $1 \mathrm{Ti}$,

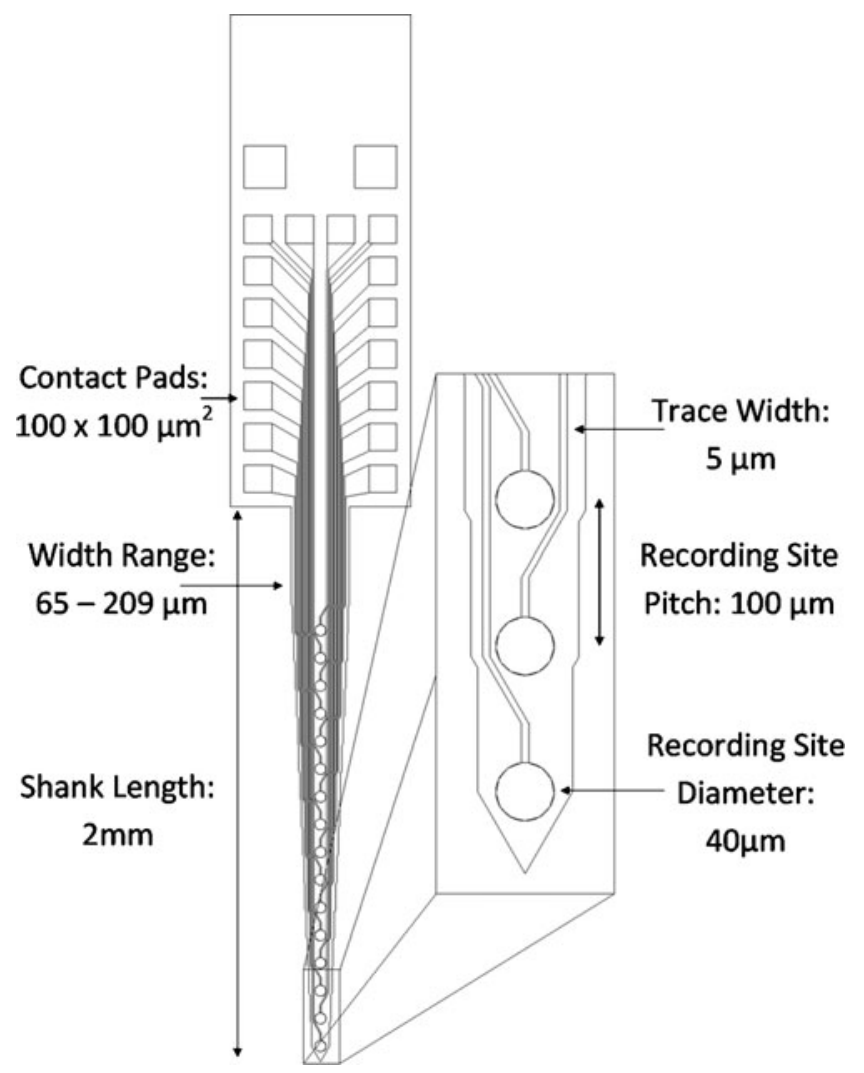

Fig. 1 Schematic representation of a typical titanium microelectrode device designed for acute cortical recording in rat

99.7\% Ti, Fine Metals Corp, Ashland, VA). The asreceived foils were first solvent cleaned by ultrasonic agitation in acetone and isopropanol, respectively, followed by DI rinsing and $\mathrm{N}_{2}$ drying. Next, as shown in Fig. 3, the first $0.6 \mu \mathrm{m} \mathrm{SiO}_{2}$ dielectric layer was deposited by plasma-enhanced chemical vapor deposition (PECVD) to insulate the subsequent electrical structures from the substrate (Benchmark 800 CVD, Axic Inc, Santa Clara, $\mathrm{CA}$; Process conditions $\mathrm{SiO}_{2}: 230 \mathrm{mT}$ chamber pressure, $26 \mathrm{~W}$ RF power, $200 \mathrm{sccm} \mathrm{N}_{2} \mathrm{O}, 35 \mathrm{sccm} 5 \% \mathrm{SiH}_{4}$, and $300^{\circ} \mathrm{C}$ lower electrode temperature). The substrates were then subjected to solvent cleaning, and mounted to $100 \mathrm{~mm}$ Si carrier wafers with thermally conductive adhesive tape (9882, 3M Electronics, St. Paul, MN). Recording sites, traces, and contact pads were then patterned via standard photolithographic liftoff techniques with electron beam depositions of $20 \mathrm{~nm} \mathrm{Ti} / 500 \mathrm{~nm}$ Au (CHA SE-600, CHA Industries, Fremont, CA; Process conditions: $1.0 \times 10^{-6} \mathrm{~T}$ base pressure and $1 \AA /$ s deposition rate).

Following metal deposition, the samples were soaked in acetone to release the Ti foils from the carrier wafer and then solvent cleaned. The second dielectric layer, composed of $0.2 \mu \mathrm{m} \mathrm{Si} \mathrm{S}_{4}$ followed by $0.8 \mu \mathrm{m}$ of $\mathrm{SiO}_{2}$, was then deposited by PECVD to provide insulation from the surrounding environment (Benchmark 800 CVD, Process 


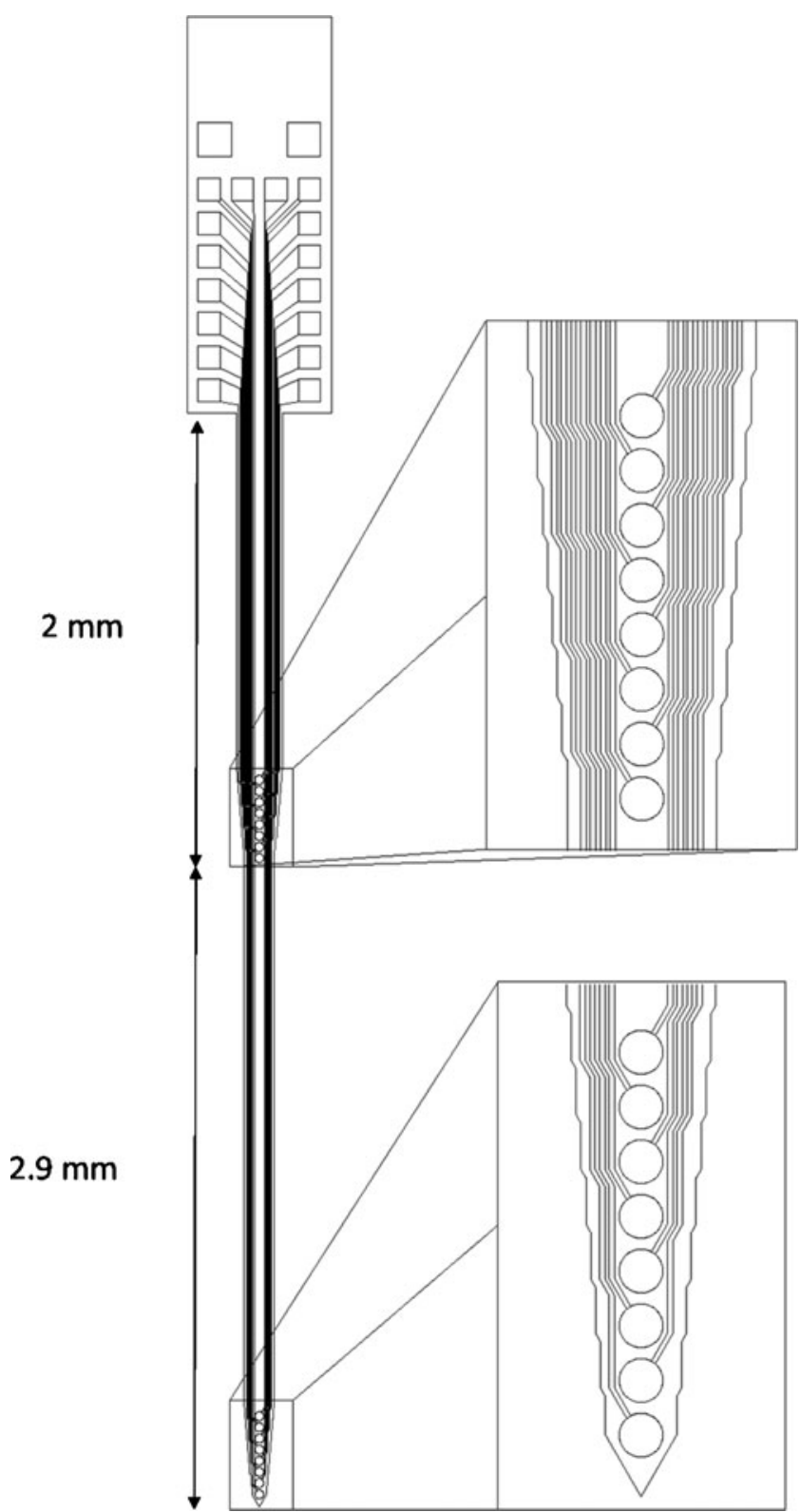

Fig. 2 Schematic representation of a typical titanium microelectrode device designed for acute auditory corticothalamic loop recording in rat

conditions $\mathrm{Si}_{3} \mathrm{~N}_{4}: 400 \mathrm{mT}$ chamber pressure, $100 \mathrm{~W} \mathrm{RF}$ power, $100 \mathrm{sccm} \mathrm{NH}_{3}, 120 \mathrm{sccm} 5 \% \mathrm{SiH}_{4}$, and $300^{\circ} \mathrm{C}$ lower electrode temperature). This dual layer stack was chosen to promote adhesion to the underlying Au patterns and minimize stress-induced curvature of the devices arising from intrinsic stresses and thermal expansion mismatch between the deposited film and the underlying Ti substrate.

After completion of the second dielectric layer deposition, the Ti foils were again mounted to $100 \mathrm{~mm}$ carrier wafers with thermally conductive tape. The contact and recording site windows were then opened via photolitho- graphic patterning and dry etching of the second dielectric layer (E620 R\&D, Panasonic Factory Solutions, Japan; Process conditions: $7.5 \mathrm{mT}$ chamber pressure, $500 \mathrm{~W}$ ICP source power, $400 \mathrm{~W}$ substrate RF power, and $40.0 \mathrm{sccm}$ $\mathrm{CHF}_{3}$ ). The photoresist was then stripped and the samples were soaked in acetone to release the Ti foils from the carriers.

The third dielectric layer, composed of $0.2 \mu \mathrm{m} \mathrm{Si}{ }_{3} \mathrm{~N}_{4}$ and $3.00 \mu \mathrm{m} \mathrm{SiO}_{2}$, was then deposited by PECVD to serve as an etch mask for the subsequent deep etch of the underlying Ti substrate. The samples were re-mounted to carrier wafers, and the shank profiles were patterned and transferred into the dielectric layers via dry etching (E620 R\&D, Process conditions: $1.9 \mathrm{mT}$ chamber pressure, $900 \mathrm{~W}$ ICP source power, $200 \mathrm{~W}$ substrate RF power, and $40.0 \mathrm{sccm} \mathrm{CHF}_{3}$ ). The photoresist was then stripped and the shank profiles were transferred through the underlying Ti substrate using the titanium inductively coupled plasma deep etch (TIDE) process (E620 R\&D, Process conditions: $15 \mathrm{mT}$ chamber pressure, $400 \mathrm{~W}$ ICP source power, $100 \mathrm{~W}$ substrate RF power, $100 \mathrm{sccm} \mathrm{Cl}$, and $5 \mathrm{sccm}$ Ar) (Parker et al. 2005). The samples were then subjected to a final short dry etch to remove the remaining thin dielectric layer protecting the contact pads and recording sites, and released from the carrier by soaking in acetone. Scanning electron micrographs of a typical completed device are shown in Fig. 4. The smooth vertical sidewalls resulting from the TIDE process are clearly evident, as is the integrity of the dielectric and metal layers deposited on the titanium substrate.

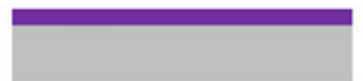

1) Deposit First Dielectric Layer

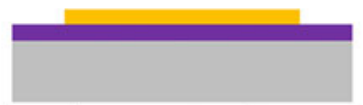

2) Mask 1: Deposit and Pattern Metal Layer

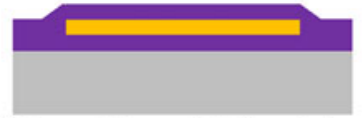

3) Deposit Second Dielectric Layer

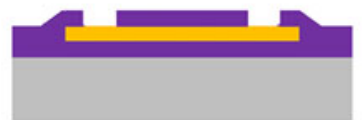

4) Mask 2: Etch Second Dielectric Layer to Open Contact Pads and Recording Sites

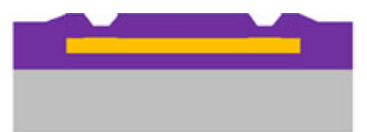

5) Deposit Dielectric Mask Layer

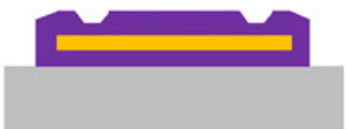

6) Mask 3: Etch Dielectric Mask Layer

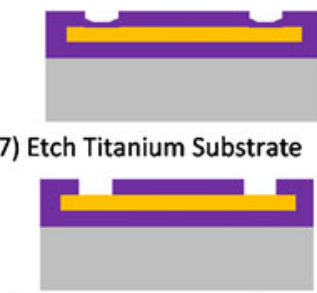

8) Reopen Contact Pads and Recording Sites

- Titanium

- Dielectric

- Gold
Fig. 3 Fabrication process flow for titanium microelectrodes 
Fig. 4 Scanning electron micrographs of titanium microelectrodes at varying magnifications

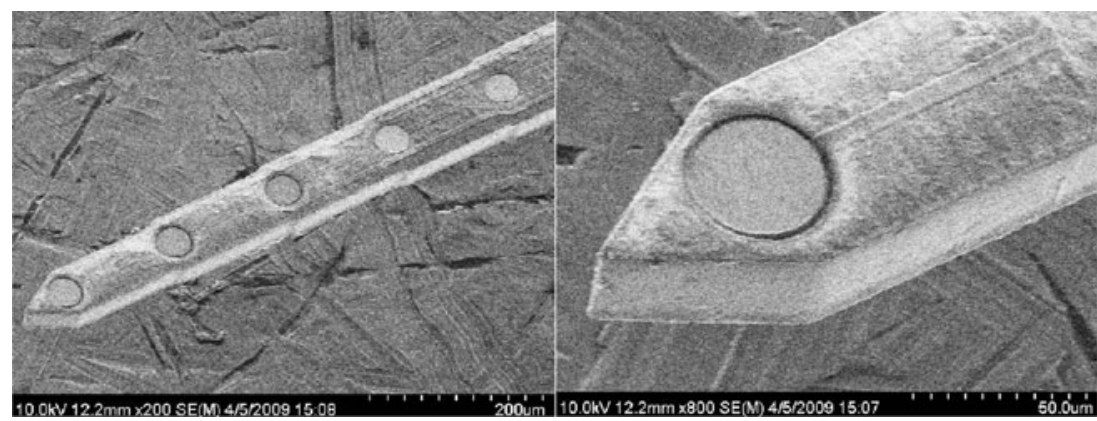

duration (Otto, Johnson and Kipke 2006, Johnson, Otto and Kipke 2005).

The microelectrode devices were packaged by bonding to commercially-available printed circuit boards (PCBs) (A16, NeuroNexus Technologies) using cyanoacrylate adhesive (Pacer Technology, Rancho Cucamonga, CA). Gold wire-bonding was then used to make connections between the contact pads on the devices and their respective bond pads on the PCBs (7400A, West-Bond, Anaheim, CA). Afterwards, an additional layer of cyanoacrylate was applied over the contact pad area as an encapsulant to protect the exposed wires.

\subsection{In situ recording site cleaning}

After device fabrication and packaging was completed, intermittent contamination of the recording sites was observed on a number of devices during optical inspection, an example of which is shown in Fig. 5(a). Although the exact nature of this contamination is not known, it is believed to be residual dielectric not cleared by the final dry etch. As shown in Fig. 6, measurements performed using the in vitro testing apparatus described in Section 3.2 indicated that this contamination led to undesirably high impedance and low charge carrying capacity. As such, most devices were subjected to a $1 \mathrm{~min}, 1.5 \mathrm{VDC}$ pulse using the in vitro testing apparatus. The resulting electrolysis at the recording sites facilitated residue removal, as evidenced by increased reflectivity of the cleaned site shown in Fig. 5(b). The cleaning process typically improved site performance, as evidenced by the reduced impedance and increased charge carrying capacity shown in Fig. 6. This in vitro recording site cleaning process is similar to those that have been performed during in vivo studies with shorter pulse

\section{Testing}

\subsection{Critical buckling force measurement}

The buckling behavior of the microelectrode devices was assessed by longitudinal uniaxial compression testing. Both titanium devices and commercially-available silicon devices (A1 ×16-5 mm 100-413, NeuroNexus Technologies) were tested, the latter for performance benchmarking purposes. The devices were mounted to a silicon carrier chip using cyanoacrylate adhesive or double-sided carbon tape. The mounted devices were then attached to a manually-driven micromanipulator (M3301R, World Precision Instruments Inc., Sarasota, FL), which was used to load the device tips against a microbalance scale (AB54-S/FACT, Mettler Toledo, Columbus, $\mathrm{OH})$. Forces exerted by the tips of the devices during testing were recorded using the microbalance (Rousche et al. 2001) and the devices were carefully observed for buckling and fracture via a CCD with magnifying optics. Five microelectrodes of the same design variant were tested for each length (i.e. Variant $3-L=2 \mathrm{~mm}$; Variant 9- $L=$ $4.9 \mathrm{~mm}$; and Variant $18-L=5.4 \mathrm{~mm}$ ).

\subsection{In vitro functional characterization}

The electrical performance of the microelectrode devices and the effect of plastic deformation on recording functionality were assessed in vitro using electrochemical impedance spectroscopy (EIS) and cyclic voltammetry (CV). In these studies, only titanium devices were tested, since silicon-
Fig. 5 Optical micrographs of recording site reflectivity: (a) before; and (b) after in vitro cleaning
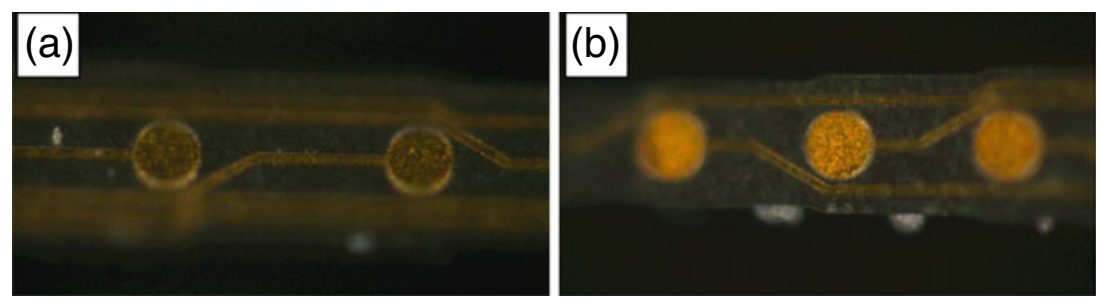

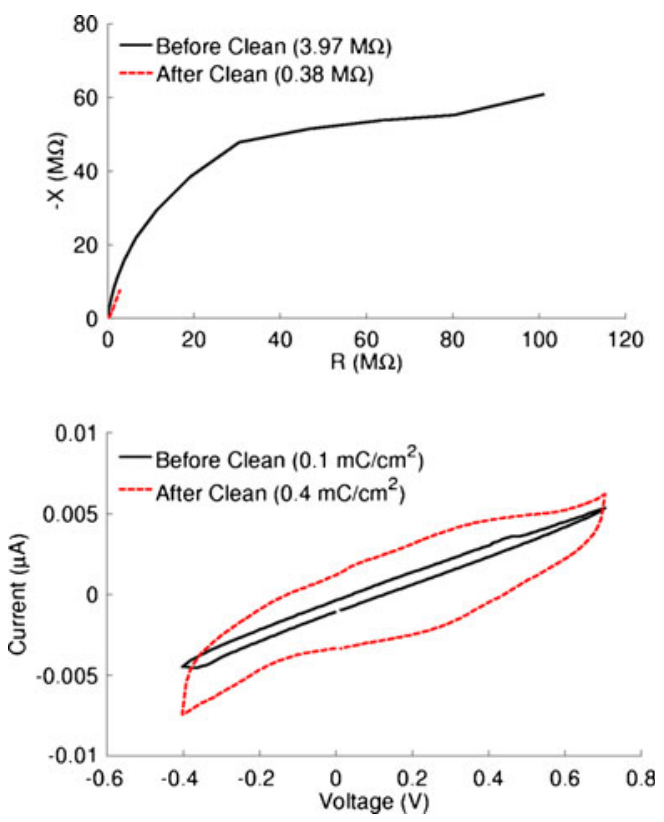

Fig. 6 Measured impedance and charge carrying capacity in a device with contaminated recording sites before and after in vitro cleaning

based devices would be expected to fail catastrophically and, therefore, lose all recording functionality. Packaged devices were tested using the three-electrode test apparatus schematically illustrated in Fig. 7. The microelectrode-bearing PCB was connected to a wiring harness attached to ribbon cabling leading to an Autolab potentiostat PGSTAT12 (EcoChemie, Utrecht, The Netherlands) with built-in frequency analyzer (Brinkmann, Westbury, NY) (Pierce et al. 2009). The wiring harness was affixed to a manually-operated manipulator that allowed variation of the distance between the microelectrode tip and the bottom of the glass beaker. A calomel electrode (Fisher Scientific, Waltham, MA) was used as the reference electrode with a platinum wire serving as the counter electrode. Measurements were taken with the microelectrode immersed in 1X phosphate-buffered saline (PBS) solution at room temperature. The test apparatus was isolated within a copper mesh "Faraday" cage (not shown in Fig. 7). A $25 \mathrm{mV}$ RMA sine wave was applied to electrode sites for EIS tests

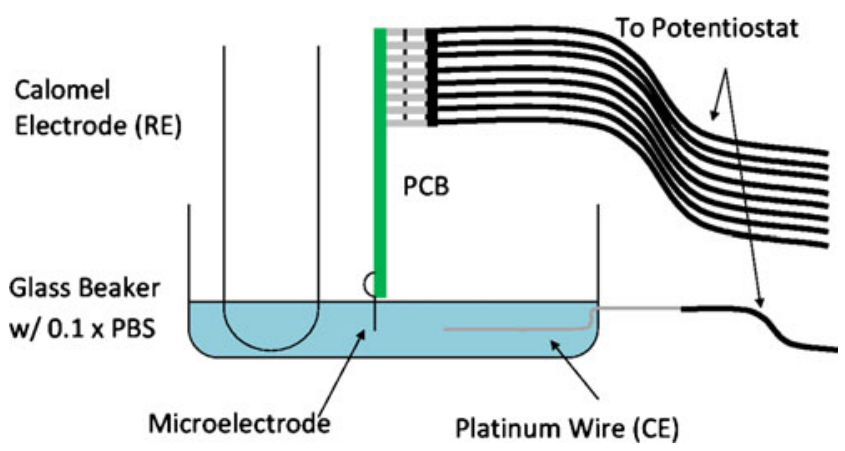

Fig. 7 Schematic of the in vitro functional characterization apparatus with frequencies ranging logarithmically from 0.1 to $10 \mathrm{kHz}$. $\mathrm{CV}$ testing was performed using a linear voltage sweep from $-0.6 \mathrm{~V}$ to $0.8 \mathrm{~V}$ with a scanning rate of $1 \mathrm{~V} / \mathrm{s}$.

Functional characterization was first performed with the microelectrode tip positioned well above the floor of the glass beaker to establish baseline device performance (i.e. Measurement \#1-Unloaded, undeformed state). Afterwards, the harness holding the PCB was manually lowered until the microelectrode tip came into contact with the bottom of the beaker, thereby imposing longitudinal uniaxial compression. The harness was then lowered further until elastic buckling was observed, at which point the harness position was fixed and EIS and CV measurements were taken again (i.e. Measurement \#2-Loaded, elastically buckled state). The harness was then further lowered until plastic deformation of the devices was induced (as verified by permanent deformation after unloading). Afterwards, the harness was raised sufficiently to fully unload the plastically-deformed device, and further EIS and CV measurements were made (i.e. Measurement \#3-Unloaded, plastically deformed state). Two to three samples of each device length were tested.

\subsection{In vivo recording}

Preliminary acute in vivo recordings were performed in a rat model. As described in greater detail elsewhere (McCarthy et al. 2011), a male Long-Evans rat was chosen, weighed, and placed under anesthesia. A midline incision was made through the scalp and the muscles were retracted. A head holder mount was constructed to stabilize the rat's skull during subsequent microelectrode insertion and testing. The skull over the primary auditory cortex of the right hemisphere was then drilled using a burr and the exposed area was cleaned and prepared for device insertion. The microelectrode array was inserted through the intact dura mater into the cortical mantle (i.e. dura was not retracted). Broadband acoustic noise stimuli were then administered and electrophysiological recording was performed through the connection of an external pre-amplifier (Tucker-Davis Technologies, Alachua, FL) connected to the microelectrode PCB. The preamplifier digitized and transmitted the neural signals to a neural recording system for amplification, analysis, and storage. All animal procedures complied with guidelines for the care and use of laboratory animals and were approved by the Purdue Animal Care and Use Committee.

\section{Results}

\subsection{Critical buckling force testing results}

The average critical buckling forces of the titanium microelectrode devices were measured to be $19.41 \pm 4.41$, 
$20.88 \pm 4.18$, and $99.80 \pm 20.70 \mathrm{mN}$ for the $5.4,4.9$, and $2.0 \mathrm{~mm}$ length devices, respectively. In contrast, the average measured elastic buckling load for five $5 \mathrm{~mm}$ shank length commercially-available silicon devices was observed to be $3.1 \pm 0.65 \mathrm{mN}$. Figure 8 plots the average buckling loads and standard deviations for titanium microelectrodes of varying designs and lengths relative to theoretical critical buckling loads, $P_{c r}$, estimated using Eulerian buckling analysis, which models the microelectrodes as long, slender columns under uniaxial longitudinal compressive loading (Rousche et al. 2001, Najafi, Ji and Wise 1990, Parker et al. 2007):

$\mathrm{P}_{\mathrm{cr}}=\frac{\pi^{2} \mathrm{EI}}{\mathrm{L}_{\mathrm{e}}^{2}}$

$\mathrm{I}=\frac{\mathrm{wt}^{3}}{12}$

where $E$ is the elastic modulus $\left(E_{S i}=166 \mathrm{GPa}\right.$ (Senturia 2004); $E_{T i}=107 \mathrm{GPa}$ (Callister 2003)), $I$ the moment of inertia, $L_{e}$ the effective shank length, $w$ the shank width, and $t$ the shank thickness. Effective shank length is dictated by the choice of end support conditions, with previous studies demonstrating fixed-free or fixed-pinned conditions as the most appropriate for the given experimental conditions (Najafi et al. 1990, Parker et al. 2007). As such, critical bucking load estimations were performed for each device length with $L_{e}=2 L$ (fixed-free) and $L_{e}=0.7 L$ (fixedpinned), where $L$ was taken to be the actual device shank length.

To simplify calculation of the theoretical critical bucking loads reported in Fig. 8, the tapered device shanks were approximated as uniform columns, i.e. columns with constant modulus, thickness, and width. The effective

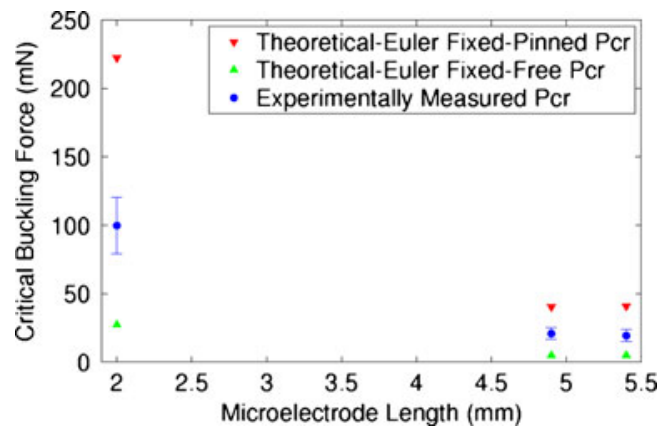

Fig. 8 Plot of experimentally measured buckling loads for various titanium microelectrode design variants vs. theoretical Euler critical buckling loads for fixed-free and fixed-pinned end conditions. Experimental data points represent the average measured buckling load for 5 specimens. The standard deviations of the reported measurements for each design variant are $20.70 \mathrm{mN}, 4.18 \mathrm{mN}$, and $4.41 \mathrm{mN}$ for shank lengths of $2 \mathrm{~mm}, 4.9 \mathrm{~mm}$, and $5.4 \mathrm{~mm}$, respectively shank width was defined to be that which was necessary to maintain equivalent planar shank area for a given shank length. The shank thickness was defined as the sum of the thicknesses of the Ti foil used for the device fabrication and the dielectric layer stack $(\sim 1.5 \mu \mathrm{m})$. Although vendor specifications for the Ti foil indicated $25.4 \mu \mathrm{m}$ nominal thickness, scanning electron microscope measurements performed on selected devices indicated actual thicknesses ranging from 34.2 to $37.6 \mu \mathrm{m}$ with an average thickness of $35.2 \mu \mathrm{m}$ (including dielectric stack), which slightly exceeds the stated manufacturing tolerance. As such, the experimentally measured average thickness was used for critical buckling load estimates. Finally, the elastic modulus of the column material was simply taken to be that of titanium, since the contribution of the dielectric layer stack would be minimized by its small thickness relative to the Ti substrate, and the relatively small modulus mismatch between $\mathrm{Ti}$ and the stack's major constituents (i.e. $\mathrm{E}_{\mathrm{SiO}_{2}}=70 \mathrm{GPa}$ (Beer, Johnston and DeWolf 2002) and $\mathrm{E}_{\mathrm{Au}}=80 \mathrm{GPa}$ (Campbell 2001)). Although not reported here, finite element analyses performed for selected design variants with true device dimensions demonstrated excellent agreement with analytical solutions, thus suggesting that the underlying simplifying assumptions used for the analytical solutions did not introduce significant error.

\subsection{In vitro functional characterization results}

Measured EIS results for the titanium devices in the unloaded, undeformed state (i.e. measurements made with the device suspended in the in vitro characterization apparatus prior to mechanical loading against the beaker floor) indicated impedance values between 0.20 and $0.43 \mathrm{M}$ $\Omega$ at $1 \mathrm{kHz}$ frequency for recording site diameters of $40 \mu \mathrm{m}$, while a range of $0.75-1.38 \mathrm{M} \Omega$ was found for recording site diameters of $23 \mu \mathrm{m}$. Measured CV results revealed maximum charge carrying capacities ranging from 0.1 to $1.9 \mathrm{mC} / \mathrm{cm}^{2}$ for all design variants.

Figure 9 shows results from EIS and CV measurements for a single $23 \mu \mathrm{m}$ recording site located near the middle of the tensile face of a $2 \mathrm{~mm}$ length titanium microelectrode device in the: a) unloaded, undeformed state; b) loaded, elastically deformed state; and c) unloaded, plastically deformed state. Increased impedance between the undeformed and deformed states suggests degradation of recording performance. Table 1 gives the average measured charge carrying capacities and impedances of ten similar $23 \mu \mathrm{m}$ recording sites from two different devices for each of the loading states. While failure to perform in situ recording site cleaning in these devices resulted in significant scatter, similar recording performance trends were observed. Moreover, while most devices were observed to buckle such that tensile stress was imposed 
EIS

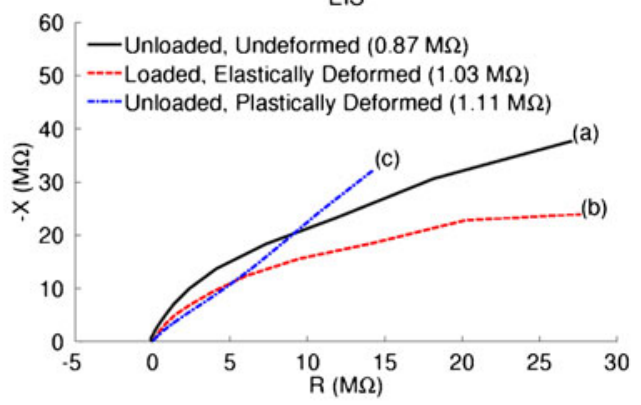

CV

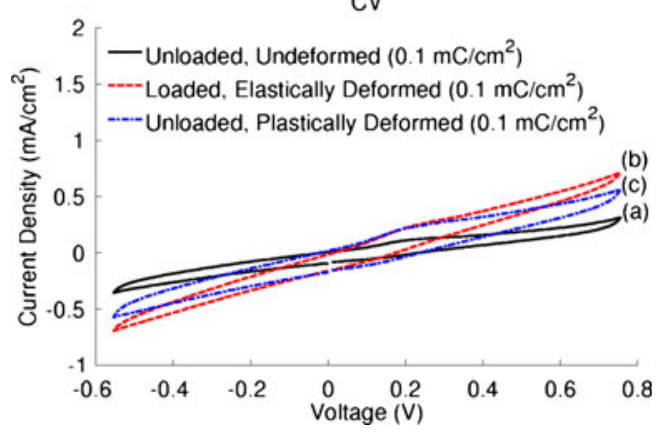

Fig. 9 Results from EIS (top) and CV (bottom) testing of a titanium microelectrode device in: (a) unloaded, undeformed state; (b) loaded, elastically deformed state; and (c) unloaded, plastically deformed state. Both EIS and CV data were taken from the same recording site, which was located near the middle of the tensile face of the device

on the dielectric stack, similar trends in performance degradation were observed for devices in which buckling resulted in imposition of compressive stress upon the dielectric stack.

Figure 10 shows scanning electron micrographs of titanium microelectrodes imaged after plastic deformation within the in vitro functional characterization apparatus for devices in which the recording sites and traces were observed to lie on the tensile face ( $\mathrm{a} \& \mathrm{~b}$ ) or the compressive face (c \& d) of the device. Although the manual control of device displacement and absence of load recording capability during these tests precluded quantitative correlation of the degree of damage with imposed load or strain, the micrographs clearly demonstrate that dielectric cracking occurred during plastic deformation of the titanium shank, as would be expected due to the low toughness of the dielectric materials. However, the micrographs indicate that despite this damage, there is minimal evidence of dielectric loss and debris generation, at least within the experimental conditions explored during the current study.

\subsection{In vivo recording results}

Despite their significant length, the titanium microelectrodes were able to penetrate the dura mater, due to an increased cross-sectional area relative to commerciallyavailable silicon-based microelectrodes. Figure 11 shows a recording trace from a single microelectrode site implanted in the auditory thalamus of an anesthetized rat (Device configuration: $5.4 \mathrm{~mm}$ shank length, $23 \mu \mathrm{m}$ diameter sites, and $100 \mu \mathrm{m}$ pitch). There was an easily isolatable action potential within the electrophysiologic data. The signal-tonoise ratio was 6.64 , which is comparable to "good" recordings from conventional silicon-based microelectrode arrays (Suner et al. 2005).

\section{Discussion}

\subsection{Buckling behavior}

Assessment of buckling behavior was performed with the primary intent of ensuring that the titanium microelectrodes possessed sufficient stiffness for penetration of rat cortex, prior to undertaking in vivo studies. This was confirmed by observation of buckling loads for all device design variants $($ range $=12.26-132.35 \mathrm{mN})$ that were well in excess of the $\sim 1 \mathrm{mN}$ insertion force reportedly required for insertion of silicon microelectrodes with comparable design into rat cortex (Jensen, Yoshida and Hofmann 2006). This, therefore, suggests that the titanium microelectrodes will be able to sustain insertion forces many times greater than those expected in vivo.

The experimental measurements suggest that the tested silicon-based devices also possess sufficient stiffness for cortical insertion, although their margin of safety is significantly reduced relative to the $\mathrm{Ti}$ devices (measured buckling load range $=1.85 \mathrm{mN}$ to $4.10 \mathrm{mN}$ ). The increased buckling resistance observed in titanium-based microelectrodes relative to the silicon-based devices can be largely explained by their increased thickness $(\sim 35 \mu \mathrm{m}$ for $\mathrm{Ti}$ devices vs. $\sim 15 \mu \mathrm{m}$ for $\mathrm{Si}$ devices), which produces significant increase in moment of inertia, due to cubic
Table 1 Average measured charge carrying capacities and impedances of $23 \mu \mathrm{m}$ recording sites during buckling testing of titanium microelectrodes $(10$ sites over 2 devices)

\begin{tabular}{lcc}
\hline Loading State & Charge Carrying Capacity $\left(\mathrm{mC} / \mathrm{cm}^{2}\right)$ & Impedance $(\mathrm{M} \Omega)$ \\
\hline Unloaded, Undeformed & $0.32 \pm 0.10$ & $1.51 \pm 0.23$ \\
Loaded, Elastically Deformed & $0.60 \pm 0.22$ & $1.83 \pm 0.53$ \\
Unloaded, Plastically Deformed & $0.53 \pm 0.28$ & $1.67 \pm 0.51$ \\
\hline
\end{tabular}


Fig. 10 Scanning electron micrographs of typical examples of dielectric damage resulting from plastic deformation of titanium microelectrodes: (a) \& (b) Typical surface morphologies near the middle of the shank of devices where the recording sites and traces were observed to lie on the tensile face; (c) \& (d) Typical surface morphologies near the middle of the shank of devices where the recording sites and traces were observed to lie on the compressive face
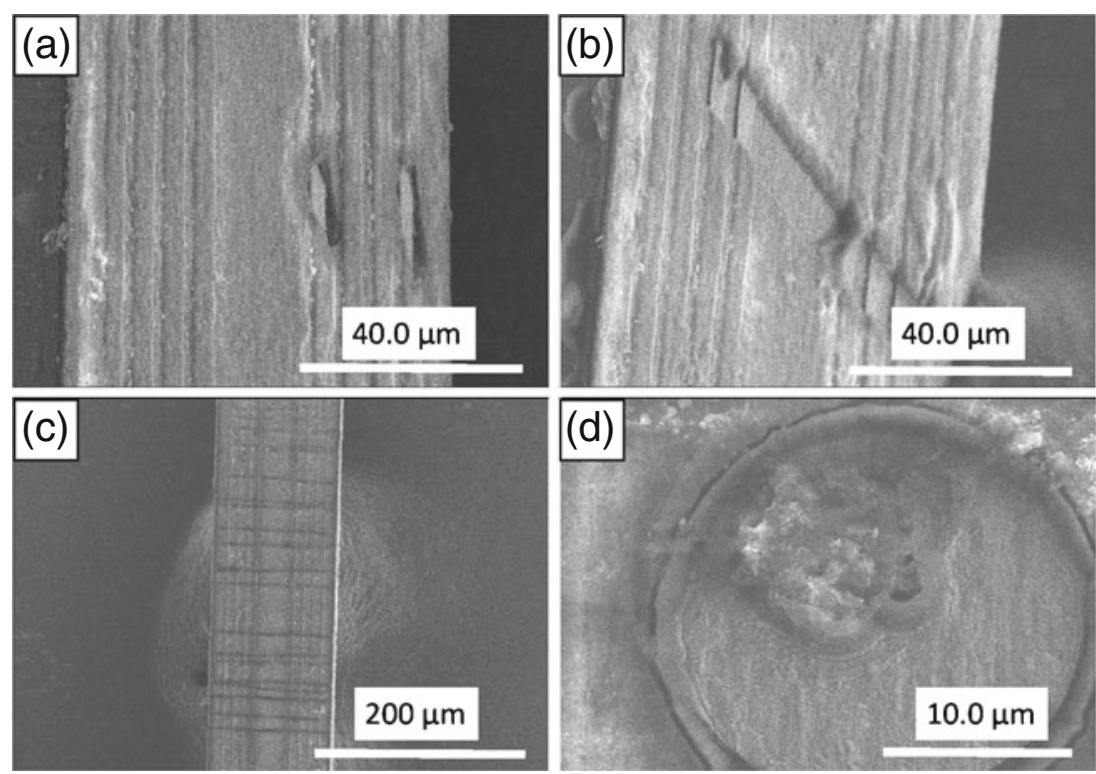

dependence on thickness (Eq. 2). However, if silicon and titanium-based devices with equivalent dimensions (i.e. $w$, $t$, and $L_{e}$ ) are compared, the following relationship emerges:

$\mathrm{P}_{\mathrm{Ti}}=\frac{\mathrm{E}_{\mathrm{Ti}}}{\mathrm{E}_{\mathrm{Si}}} \mathrm{P}_{\mathrm{Si}}$

As can be seen, the critical buckling loads for titanium and silicon-based devices can be related through the ratio of their elastic moduli. Substitution of appropriate moduli values into Eq. 3 reveals that the predicted critical buckling load for a titanium microelectrode would be approximately $66 \%$ of a comparable silicon device.

While reduced buckling resistance implies reduced insertion performance relative to silicon, the observation of graceful failure via plastic deformation indicates potential for additional load bearing capacity beyond the onset of elastic buckling, thus forestalling fracture, often significantly. This is evidenced in the current study, as well a prior study of titanium microneedles with similar geometry (Parker et al. 2007), by the absence of fracture-based failure in any

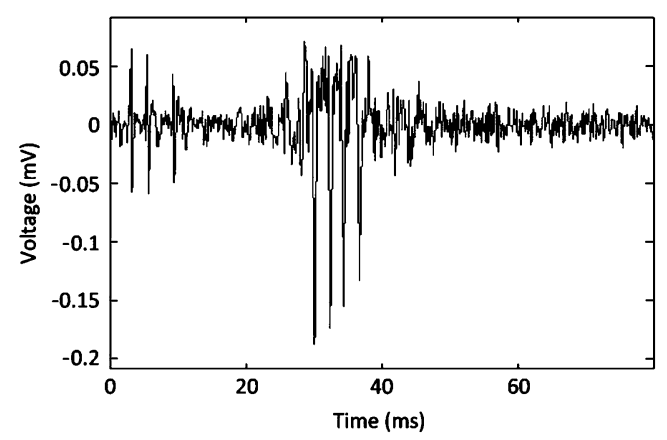

Fig. 11 Extracellular recording from auditory thalamus of an anesthetized rat titanium device, even those tested well beyond the elastic buckling limit. The intrinsic brittleness of silicon, in contrast, largely precludes plasticity, thus resulting in onset of fracture soon after the elastic buckling limit has been exceeded, as was observed during testing of the silicon devices in the current study. As such, titanium microelectrodes provide greater tolerance to overloading during insertion and largely preclude potential for catastrophic fragmentation within the brain, thus increasing potential for enhanced reliability and safety relative to conventional silicon devices.

The data presented in Fig. 8 suggest that neither fixedpinned nor fixed-free end conditions accurately model the experimentally measured buckling loads for the titanium microelectrode devices, although there is slight bias towards the latter. This may be partly explained by potential for minor slippage between the device tip and the smooth surface of the silicon chip $\left(\mathrm{R}_{\mathrm{A}}<1 \mathrm{~nm} \mathrm{RMS}\right)$ and/or the chip and the microbalance plate. While there were no observations of gross tip slippage during testing, the limited spatial resolution of the imaging system may have precluded observation of less pronounced slippage. Furthermore, manual control of device displacement during testing reduced the accuracy with which the critical buckling load could be determined, thus introducing potential for additional error. However, despite these limitations, the measured data demonstrate that the analytical solutions (and their underlying simplifying assumptions) can be used to estimate the bounds within which buckling is expected, at least within the design space considered in the current study.

\subsection{In vitro recording behavior}

Assessment of electrical performance was primarily performed with the intent of ensuring that the titanium devices 
provided sufficient recording performance, prior to undertaking in vivo studies. This was confirmed by measurement of impedances for devices in the unloaded, undeformed state $(40 \mu \mathrm{m}$ diameter site range $=0.20-0.43 \mathrm{M} \Omega ; 23 \mu \mathrm{m}$ diameter site range $=0.75-1.38 \mathrm{M} \Omega$ ) that were in good agreement with specified values for silicon-based commercial devices (range $=0.5-3.0 \mathrm{M} \Omega$, depending on the recording site diameter (Paralikar and Clement 2008)). Similarly, measured charge carrying capacities (range = $0.1-1.9 \mathrm{mC} / \mathrm{cm}^{2}$ ) were observed to be in fair agreement with silicon devices reported in the literature which utilize gold recording sites of similar sizes (Wise et al. 2004).

A secondary intent of the in vitro characterization studies was to determine whether titanium microelectrodes were able to maintain recording functionality after being subjected to elastic buckling or plastic deformation. The inability to accurately measure either load or displacement during these studies precluded systematic and quantitative analysis of the effect of deformation on recording performance. However, the data shown in Fig. 9 qualitatively demonstrate that titanium microelectrodes are able to retain a significant portion of their original recording functionality during elastic buckling, as well as after plastic deformation and unloading. Silicon devices were not tested in these studies, but it is reasonable to expect that their brittleness would severely limit possibility for continued recording functionality beyond elastic buckling, since fracture would soon follow. As such, this suggests that titanium microelectrodes may provide greater recording reliability relative to silicon devices, since some level of recording functionality can be retained despite overloading.

Definitive elucidation of the mechanisms underlying the degradation of recording performance with device deformation is beyond the scope of the current study. However, the scanning electron micrographs shown in Fig. 10 suggest that low dielectric fracture toughness results in accumulation of damage within the dielectric stack, e.g. cracking or delamination. Concomitant damage to the gold traces and recording sites would also be expected, e.g. plasticity, cracking, and/or delamination from the dielectric at severe strains. These types of damage would be expected to have increasingly detrimental effect on the recording performance. Although the observation of increased impedance with deformation supports this presumption, further study is required for validation. Nevertheless, when coupled with the plastic failure mode of the underlying titanium shank, the observed retention of the damaged dielectric suggests potential for an added degree of safety during insertion, relative to silicon-based devices. The degree of deformation required to produce such damage would be immediately apparent during the insertion attempt and would most likely occur prior to actual penetration. As such, opportunity would be provided for unloading and removal of the damaged device with minimal danger of fragmentation or debris generation, thus minimizing potential for adverse effect upon surrounding neural tissue, aside from trauma associated with compressive dimpling of the cortical surface during the attempted insertion. This stands in contrast to brittle silicon-based devices, whose failure under buckling presents potential for generation of numerous atomically-sharp fragments, both large and small.

Finally, although less likely, it is conceivable that titanium-based devices could be subjected to mechanical overloading once inserted. In such cases, dielectric cracking could result in eventual debris generation within the brain, due to potential for delamination produced by penetration of physiological fluids into the cracks. While the magnitude and severity of such debris would be far less than that associated with fracture of a comparable silicon-based device, this represents a potential concern nonetheless. Replacement of the current brittle dielectrics with ductile polymers could provide one means for addressing this concern. Polyimide represents an attractive candidate in this regard, due to its demonstrated biocompatibility in neural implantation applications (Richardson, Miller and Reichert 1993, Stieglitz and Meyer 1999, Rubehn and Stieglitz 2010). The use of polyimide as the structural basis for neural prostheses (Rousche et al. 2001, Stieglitz and Gross 2001), as well as flexible cabling for such devices (Hetke, Najafi and Wise 1990), has been demonstrated. However, the coupling of polyimide with titanium (as the primary structural component) would offer means for improving insertion reliability and recording site placement accuracy while minimizing shank cross section area.

\subsection{In vivo recording}

Assessment of in vivo performance was primarily performed with the intent of validating cortical penetration capability of the titanium microelectrodes, as well their acute neural recording capability. The preliminary in vivo results confirmed that titanium microelectrodes possessed sufficient stiffness to penetrate not only rat pia, but also dura, which is typically retracted prior to insertion of commerciallyavailable silicon devices. Moreover, initial in vivo studies generated isolatable action potentials with signal to noise ratios comparable to commercially-available silicon-based devices. Subsequent studies have also demonstrated capability for simultaneous acute recording from both auditory cortex and thalamus from the same microelectrode (McCarthy et al. 2011). To the best of our knowledge, this represents the first such measurement made using a single microelectrode device. As discussed earlier, this capability may provide significant opportunity for facilitating studies targeted towards enhancing understanding of sequentially interconnected neural processing networks. 
While these results are promising, chronic implantation studies are needed to assess long-term device stability and recording reliability. Such studies may highlight need for consideration of additional or alternative dielectric materials, such as amorphous silicon carbide and silicon oxycarbide, which have been shown to provide superior chemical stability and impermeability relative to $\mathrm{SiO}_{2}$ and $\mathrm{Si}_{3} \mathrm{~N}_{4}$ in physiological saline environments (Cogan et al. 2003). They may also demonstrate need for consideration of recording site coatings, such as sputtered iridium oxide, which have been shown to provide significantly lower impedance than noble metals (Cogan, Plante and Ehrlich 2004). Finally, and perhaps most importantly, such studies are also likely to demonstrate need for integration of means for mitigating reactive tissue response (i.e. gliosis), which often degrades recording quality with time (Williams, Rennaker and Kipke 1999, Vetter et al. 2004, Williams et al. 2007). Use of titanium rather than silicon as the structural backbone for microelectrode devices is unlikely to provide benefit in this regard, since such substitution does not address the presumed mechanisms underlying reactive tissue response, i.e. implantation-induced injury and/or mechanical impedance mismatch with the surrounding tissue. However, numerous strategies have been proposed within this context, including pharmacological intervention (Shain et al. 2003, Kim and Martin 2006, Zhong and Bellamkonda 2007), surface modification (Wadhwa, Lagenaur and Cui 2006, Moxon et al. 2004a, Abidian and Martin 2009), and novel device design (Seymour and Kipke 2007, Kipke et al. 2008, Muthuswamy et al. 2005). The unique capabilities afforded by titanium micromachining, coupled with the favorable properties of titanium, may prove particularly advantageous for implementation of such strategies.

\section{Conclusion}

We have reported the design, fabrication, mechanical testing, in vitro, and in vivo characterization of titaniumbased, "Michigan" type, penetrating microelectrodes for acute neural recording in rat. Mechanical testing results demonstrate that these devices posses sufficient stiffness for reliable cortical penetration, and also demonstrate that they provide potential for greater mechanical reliability and safety relative to current commercially-available silicon devices, due to their graceful, plasticity-based failure mode. In vitro characterization studies demonstrate that the titanium devices provide recording performance comparable to that of silicon-based devices, and also demonstrate that they provide potential for greater recording reliability, since plasticity-based failure allows maintenance of a significant degree of recording functionality despite overloading. Finally, results from preliminary in vivo studies corroborate many of these findings by demonstrating capability for reliable cortical insertion and acute recording with good signal to noise. Collectively, these results demonstrate the potential embodied in titanium as a promising new structural material for penetrating microelectrodes.

Acknowledgment The authors would like to thank the Center for Neural Communication Technology for providing the silicon microelectrodes (NIH/NIBIB P41-EB002030). The authors also thank the staff at the Birck Nanotechnology Center (Purdue University) for assistance in device fabrication, and R. Madangopal and the students of the NeuroProstheses Research Laboratory (Purdue University) for assistance with in vitro characterization. Finally, the authors acknowledge support provided by the Cordier Fellowship Program (School of Mechanical Engineering, Purdue University), the Purdue Research Foundation, and the Showalter Research Trust (Purdue University).

Open Access This article is distributed under the terms of the Creative Commons Attribution Noncommercial License which permits any noncommercial use, distribution, and reproduction in any medium, provided the original author(s) and source are credited.

\section{References}

M.R. Abidian, D.C. Martin, Multifunctional nanobiomaterials for neural interfaces. Adv Funct Mater 19, 573-585 (2009)

M.F. Aimi, M.P. Rao, N.C. Macdonald, A.S. Zuruzi, D.P. Bothman, High-aspect-ratio bulk micromachining of titanium. Nat Mater 3, 103-105 (2004)

M.J. Bak, J.P. Girvin, F.T. Hambrecht, C.V. Kufta, G.E. Loeb, E.M. Schmidt, Visual sensations produced by intracortical microstimulation of the human occipital cortex. Med Biol Eng Comput 28, 257-259 (1990)

F.P. Beer, E.R. Johnston, J.T. DeWolf, Mechanics of materials (McGraw-Hill, New York, 2002)

W.D. Callister, Materials science and engineering: An introduction (Wiley, New York, 2003)

S.A. Campbell, The science and engineering of microelectronic fabrication (Oxford University Press, New York, 2001)

H.-Y. Chan, D. Aslam, J. Wiler, B. Casey, A novel diamond microprobe for neuro-chemical and electrical recording in neural prosthesis. J Microelectromech S 18, 12 (2009)

S.F. Cogan, D.J. Edell, A.A. Guzelian, Y.P. Liu, R. Edell, Plasmaenhanced chemical vapor deposited silicon carbide as an implantable dielectric coating. J Biomed Mater Res A 67A, 856-867 (2003)

S.F. Cogan, T.D. Plante, J. Ehrlich, Sputtered iridium oxide films (SIROFs) for low-impedance neural stimulation and recording electrodes. Conference Proceedings of the IEEE Engineering in Medicine and Biology Society 6, 4153-6 (2004)

E.E. Fanselow, M.A.L. Nicolelis, Behavioral modulation of tactile responses in the rat somatosensory system. J Neurosci 19, 76037616 (1999)

T.A. Fofonoff, S.M. Martel, N.G. Hatsopoulos, J.P. Donoghue, I.W. Hunter, Microelectrode array fabrication by electrical discharge machining and chemical etching. IEEE Trans Biomed Eng 51, 890-895 (2004)

H. Gelbard-Sagiv, R. Mukamel, M. Harel, R. Malach, I. Fried, Internally generated reactivation of single neurons in human hippocampus during free recall. Science 322, 96-101 (2008) 
J.F. Hetke, K. Najafi, K.D. Wise, Flexible miniature ribbon cables for long-term connection to implantable sensors. Sensor Actuat APhys 23, 999-1002 (1990)

L.R. Hochberg, M.D. Serruya, G.M. Friehs, J.A. Mukand, M. Saleh, A.H. Caplan, A. Branner, D. Chen, R.D. Penn, J.P. Donoghue, Neuronal ensemble control of prosthetic devices by a human with tetraplegia. Nature 442, 164-171 (2006)

D.H. Hubel, T.N. Wiesel, Laminar and columnar distribution of geniculo-cortical fibers in macaque monkey. J Comp Neurol 146, 421-450 (1972)

W. Jensen, K. Yoshida, U.G. Hofmann, In-vivo implant mechanics of flexible, silicon-based ACREO microelectrode arrays in rat cerebral cortex. IEEE Trans Biomed Eng 53, 934-940 (2006)

M.D. Johnson, K.J. Otto, D.R. Kipke, Repeated voltage biasing improves unit recordings by reducing resistive tissue impedances. IEEE Trans Neural Syst Rehabil Eng 13, 160-165 (2005)

D.H. Kim, D.C. Martin, Sustained release of dexamethasone from hydrophilic matrices using PLGA nanoparticles for neural drug delivery. Biomaterials 27, 3031-3037 (2006)

D.R. Kipke, W. Shain, G. Buzsaki, E. Fetz, J.M. Henderson, J.F. Hetke, G. Schalk, Advanced neurotechnologies for chronic neural interfaces: New horizons and clinical opportunities. J Neurosci 28, 11830-11838 (2008)

K. Lee, J. He, R. Clement, S. Massia, B. Kim, Biocompatible benzocyclobutene (BCB)-based neural implants with microfluidic channel. Biosens Bioelectron 20, 404-407 (2004a)

K.K. Lee, J.P. He, A. Singh, S. Massia, G. Ehteshami, B. Kim, G. Raupp, Polyimide-based intracortical neural implant with improved structural stiffness. J Micromech Microeng 14, 32-37 (2004b)

P.T. McCarthy, R. Madangopal, K.J. Otto, M.P. Rao. Titanium-based multi-channel, micro-electrode array for recording neural signals. In 31st Annual International Conference of the IEEE Engineering in Medicine \& Biology Conference. Minneapolis, MN, 2009

P.T. McCarthy, M.P. Rao, K.J. Otto. Simultaneous recording of rat auditory cortex and thalamus via a titanium-based multi-nucleus, microelectrode array. submitted to Journal of Neural Engineering (2011)

P.S. Motta, J.W. Judy, Multielectrode microprobes for deep-brain stimulation fabricated with a customizable 3-D electroplating process. IEEE Trans Biomed Eng 52, 923-933 (2005)

K.A. Moxon, N.M. Kalkhoran, M. Markert, M.A. Sambito, J.L. McKenzie, J.T. Webster, Nanostructured surface modification of ceramic-based microelectrodes to enhance biocompatibility for a direct brain-machine interface. IEEE Trans Biomed Eng 51, 881889 (2004a)

K.A. Moxon, S.C. Leiser, G.A. Gerhardt, K.A. Barbee, J.K. Chapin, Ceramic-based multisite electrode arrays for chronic singleneuron recording. IEEE Trans Biomed Eng 51, 647-656 (2004b)

J. Muthuswamy, M. Okandan, A. Gilletti, M.S. Baker, T. Jain, An array of microactuated microelectrodes for monitoring singleneuronal activity in rodents. IEEE Trans Biomed Eng 52, 1470 1477 (2005)

K. Najafi, J. Ji, K.D. Wise, Scaling limitations of silicon multichannel recording probes. IEEE Trans Biomed Eng 37, 1-11 (1990)

M.A. Nicolelis, M. Shuler, Thalamocortical and corticocortical interactions in the somatosensory system. Prog Brain Res 130, 90-110 (2001)

M.A.L. Nicolelis, L.A. Baccala, R.C.S. Lin, J.K. Chapin, Sensorimotor encoding by synchronous neural ensemble activity at multiple levels of the somatosensory system. Science 268, 1353-1358 (1995)

R.A. Normann, Technology insight: Future neuroprosthetic therapies for disorders of the nervous system. Nat Clin Pract Neurol 3, 444-452 (2007)
K.J. Otto, P.J. Rousche, D.R. Kipke, Cortical microstimulation in auditory cortex of rat elicits best-frequency dependent behaviors. J Neural Eng 2, 42-51 (2005a)

K.J. Otto, P.J. Rousche, D.R. Kipke, Microstimulation in auditory cortex provides a substrate for detailed behaviors. Hear Res 210 , $112-117(2005 b)$

K.J. Otto, M.D. Johnson, D.R. Kipke, Voltage pulses change neural interface properties and improve unit recordings with chronically implanted microelectrodes. IEEE Trans Biomed Eng 53, 333 340 (2006)

S.R. Otto, R.V. Shannon, E.P. Wilkinson, W.E. Hitselberger, D.B. McCreery, J.K. Moore, D.E. Brackmann, Audiologic outcomes with the penetrating electrode auditory brainstem implant. Otol Neurotol 29, 1147-1154 (2008)

K.J. Paralikar, R.S. Clement, Collagenase-aided intracortical microelectrode array insertion: Effects on insertion force and recording performance. IEEE Trans Biomed Eng 55, 22582267 (2008)

E.R. Parker, B.J. Thibeault, M.F. Aimi, M.P. Rao, N.C. MacDonald, Inductively coupled plasma etching of bulk titanium for MEMS applications. J Electrochem Soc 152, C675-C683 (2005)

E.R. Parker, M.P. Rao, K.L. Turner, C.D. Meinhart, N.C. MacDonald, Bulk micromachined titanium microneedles. J Microelectromechanic Sys 16, 289-295 (2007)

A.L. Pierce, S. Sommakia, J.L. Rickus, K.J. Otto, Thin-film silica solgel coatings for neural microelectrodes. J Neurosci Meth $\mathbf{1 8 0}$ 106-110 (2009)

R.R. Richardson, J.A. Miller, W.M. Reichert, Polyimides as biomaterials: Preliminary biocompatibility testing. Biomaterials 14, 627-635 (1993)

P. Rousche, D. Pellinen, D.J. Pivin, J. Williams, R. Vetter, D. Kipke, Flexible polyimide-based intracortical electrode arrays with bioactive capability. IEEE Trans Biomed Eng 48, 361-371 (2001)

B. Rubehn, T. Stieglitz. In vitro evaluation of the long-term stability of polyimide as a material for neural implants. Biomaterials 31 (2010)

M. Schuettler, S. Stiess, B.V. King, G.J. Suaning, Fabrication of implantable microelectrode arrays by laser cutting of silicone rubber and platinum foil. J Neural Eng 2, S121-S128 (2005)

S.D. Senturia, Microsystem design (Springer, New York, 2004)

J.P. Seymour, D.R. Kipke, Neural probe design for reduced tissue encapsulation in CNS. Biomaterials 28, 3594-3607 (2007)

W. Shain, L. Spataro, J. Dilgen, K. Haverstick, S. Retterer, M. Isaacson, M. Saltzman, J.N. Turner, Controlling cellular reactive responses around neural prosthetic devices using peripheral and local intervention strategies. IEEE Trans Neural Syst Rehabil Eng 11, 186-188 (2003)

S.M. Sherman, R.W. Guillery, The role of the thalamus in the flow of information to the cortex. Philos T Roy Soc B 357, 1695-1708 (2002)

T. Stieglitz, M. Gross. Flexible BIOMEMS with electrode arrangements on front and back side as key component in neural prostheses and biohybrid systems. Transducers '01: Eurosensors Xv, Digest of Technical Papers, Vols 1 and 2, 358-361 (2001)

T. Stieglitz, J.U. Meyer, Implantable microsystems. Polyimide-based neuroprostheses for interfacing nerves. Med Device Technol 10, 28-30 (1999)

T. Stieglitz, H. Beutel, M. Schuettler, J.U. Meyer, Micromachined, polyimide-based devices for flexible neural interfaces. Biomed Microdevices 2, 283-294 (2000)

S. Suner, M.R. Fellows, C. Vargas-Irwin, G.K. Nakata, J.P. Donoghue, Reliability of signals from a chronically implanted, silicon-based electrode array in non-human primate primary motor cortex. IEEE Trans Neural Syst Rehabil Eng 13, 524-541 (2005) 
T. Suzuki, K. Mabuchi, S. Takeuchi. A 3D flexible parylene probe array for multichannel neural recording. In Neural Engineering, 2003. Conference Proceedings. First International IEEE EMBS Conference on, 154-156 (2003)

D.H. Szarowski, M.D. Andersen, S. Retterer, A.J. Spence, M. Isaacson, H.G. Craighead, J.N. Turner, W. Shain, Brain responses to micro-machined silicon devices. Brain Res 983, 23-35 (2003)

S. Takeuchi, T. Suzuki, K. Mabuchi, H. Fujita, 3D flexible multichannel neural probe array. J Micromech Microeng 14, 104-107 (2004)

S. Takeuchi, D. Ziegler, Y. Yoshida, K. Mabuchi, T. Suzuki, Parylene flexible neural probes integrated with microfluidic channels. Lab Chip 5, 519-523 (2005)

R.J. Vetter, J.C. Williams, J.F. Hetke, E.A. Nunamaker, D.R. Kipke, Chronic neural recording using silicon-substrate microelectrode arrays implanted in cerebral cortex. IEEE Trans Biomed Eng 51, 896-904 (2004)

R. Wadhwa, C.F. Lagenaur, X.T. Cui, Electrochemically controlled release of dexamethasone from conducting polymer polypyrrole coated electrode. J Control Release 110, 531-541 (2006)
M.P. Ward, P. Rajdev, C. Ellison, P.P. Irazoqui, Toward a comparison of microelectrodes for acute and chronic recordings. Brain Res 1282, 183-200 (2009)

J.C. Williams, R.L. Rennaker, D.R. Kipke, Long-term neural recording characteristics of wire microelectrode arrays implanted in cerebral cortex. Brain Res Protoc 4, 303-313 (1999)

J.C. Williams, J.A. Hippensteel, J. Dilgen, W. Shain, D.R. Kipke, Complex impedance spectroscopy for monitoring tissue responses to inserted neural implants. J Neural Eng 4, 410-423 (2007)

J.A. Winer, D.T. Larue, Patterns of reciprocity in auditory thalamocortical connections-Study with horseradish-peroxidase and autoradiographic methods in the rat medial geniculate-body. J Comp Neurol 257, 282-315 (1987)

K.D. Wise, D.J. Anderson, J.F. Hetke, D.R. Kipke, K. Najafi, Wireless implantable microsystems: High-density electronic interfaces to the nervous system. Proc IEEE 92, 76-97 (2004)

Y.H. Zhong, R.V. Bellamkonda, Dexamethasone-coated neural probes elicit attenuated inflammatory response and neuronal loss compared to uncoated neural probes. Brain Res 1148, 15-27 (2007) 